\title{
Controversies in the medical management of breast cancer
}

\author{
Ian E. Smith \\ Department of Medicine. Rolal Marsden Hospital, Fulham Road. London. SW3. UK.
}

\section{Introduction}

Breast cancer is more of a medical than a surgical disease. Most patients presenting with clinically localised tumour sooner or later develop metastases from which they will die, and this occurs irrespective of the nature or intensity of their initial local treatment. In other words, breast cancer is usually a systemic disease from its clinical outset.

Both endocrine therapy and cytotoxic chemotherapy are widely used in the treatment of breast cancer; indeed medically there are more therapeutic options available than for any other malignancy. Yet for the patient with overt clinical metastases these are only transiently effective and virtually never curative. This limitation of medical treatment has led to two large areas of controversy. First, how best should the various forms of endocrine therapy and cytotoxic chemotherapy be put together to achieve maximum palliative benefit in patients with advanced metastatic disease? Second, might endocrine therapy or chemotherapy have the potential for cure or prolongation of survival if used early in the course of the disease as an adjuvant to surgery at a time when metastases are clinically undetectable?

\section{Treatment of advanced metastatic disease}

Treatment options: is treatment always necessary?

There are three main treatment options for the patient with metastatic breast cancer. The first two, endocrine therapy and chemotherapy, are well recognised. The third, no specific treatment at all if the disease is asymptomatic, is too frequently overlooked.

The option of no specific treatment is based on the extraordinary heterogeneity of breast cancer. Although some tumours pursue a fulminating and rapidly fatal course others progress almost imperceptively over a period of many years. Bloom et al. (1962) reviewed the case histories of 250 patients with untreated breast cancer at the Middlesex Hospital between 1805 and 1933. Nearly all had advanced

Ian E. Smith, M.D., F.R.C.P. disease. The mean survival was 2.7 years, but $18 \%$ remained alive 5 years after their diagnosis, $3.6 \%$ at 10 years, and $0.8 \%$ at 15 years. Likewise, in a more recent study Brinkley \& Haybittle (1975) described patients dying of breast cancer more than 20 years after their diagnosis, or dying of other causes but with breast cancer still present at post mortem.

These observations suggest that some patients with slow tempo disease may do just as well off treatment as on treatment for long periods if their disease is asymptomatic. Indeed the possibility is raised that toxic therapies in such patients may actually do more harm than good (see below).

\section{Endocrine therapy or chemotherapy?}

Endocrine therapy by whatever means consistently achieves tumour regression in little more than $30 \%$ of unselected patients. In contrast responses are seen in around $60 \%$ patients treated with combination chemotherapy. Because of this, and because of dramatic achievements with combination chemotherapy against other tumours including Hodgkin's disease and malignant teratoma, a vogue developed in the middle 1970s for the use of chemotherapy in preference to endocrine therapy as first line management for metastatic breast cancer. Today (for several reasons) this approach is less widely advocated, except perhaps in the USA.

First, the toxicity and side effects associated with cytotoxic drugs make this treatment more unpleasant for most patients than endocrine therapy. Second, the median response duration with chemotherapy is little more than 6-9 months and few patients sustain a response for more than a year (Henderson \& Canellos, 1980). In contrast, the median duration of response to endocrine treatment is around 12-15 months, and remissions lasting several years are sometimes seen (Kiang. 1981). In addition further remissions with second line endocrine treatment can be achieved in up to $50 \%$ of patients who have relapsed after first line therapy. Finally, the figure of $30 \%$ for responses to endocrine therapy may underestimate the total number of patients achieving benefit with this treatment: a further $15-20 \%$ patients achieve disease stabilization 
albeit without objective regression, but associated with relief of symptoms. In our experience the prognosis for these patients is almost as good as that for patients achieving tumour regression, suggesting that nearly $50 \%$ of patients may achieve benefit from endocrine treatment (Harris et al., 1982).

Some years ago a control randomised trial was carried out comparing endocrine therapy with chemotherapy in an attempt to establish which treatment was the more effective (Priestman et al., 1977). Today this approach would generally be considered naive. First, it takes no account of the inherent heterogeneity of breast cancer and the possibility that the choice of treatment may vary for different patients. Several clear-cut clinical parameters exist influencing the likelihood of response to endocrine therapy (Table I). Thus, the 70 year old with slowly progressive soft tissue metastases developing many years after her primary tumour has a very good chance of responding to simple endocrine treatment, whereas the 35 year old with fulminating liver metastases 6 months after her original diagnosis will almost certainly not respond and needs immediate chemotherapy. Second, the 'endocrine therapy or chemotherapy' argument takes no account of the possibility that both forms of treatment may be appropriate for many patients in different stages in their disease. For many patients therefore the correct question is not which form of treatment should be used but how best these two forms of therapy should be combined as discussed below.

\section{Choice of endocrine therapy}

Endocrine therapy for breast cancer has recently undergone a quiet revolution. The traditional forms of therapy using surgical ablation, oestrogens and androgens are largely being replaced by simple medical treatments which are easy to administer, and readily reversible if ineffective. The most important of these is the anti-oestrogen tamoxifen which is now widely and appropriately used as first-line endocrine treatment. There is little that is controversial here except perhaps in premenopausal patients where oophorectomy is still advocated by many as first-line treatment. Yet tamoxifen achieves a very similar

Table I Favourable predictive factors for response to endocrine therapy.

\footnotetext{
Age: older patients

Sites of disease: soft tissue, bone, pleura

Long tumour free interval

Response to previous endocrine therapy

Cytoplasmic oestrogen/progesterone receptor positive
}

response rate and response duration to oophorectomy with all the advantages of ready reversibility and ease of administration (Margreiter \& Wiegele, 1984; ${ }^{\circ}$ Kiang, 1981). Current comparative randomised trials are soon likely to confirm the clinical impressiono already strongly felt by many that tamoxifen and noto oophorectomy is now the first-line treatment of choice in premenopausal patients.

\section{Chemotherapy in advanced breast cancer}

Some general and fairly non-controversial statements. $\overrightarrow{.}$ can be made about chemotherapy in advanced breast $\vec{\omega}$ cancer. Combinations achieve higher response rateso than single agents and for this reason are usuallyo preferred (although the evidence that increased res- 3 . ponse rates are translated into survival benefit is by nog means clear-cut, as will be discussed below). Ad-riamycin is the most active single agent, but this agent $\overrightarrow{\mathrm{N}}$ causes severe alopecia and it is doubtful whetheradriamycin-containing regimens are significantly $\vec{v}$ more effective than those without this drug (Hender-으 son \& Canellos, 1980). In contrast to endocrinetherapy, there are few clear-cut clinical factors or ${ }_{\mathbb{D}}^{\top}$ laboratory tests to predict the likelihood of response to음 chemotherapy and this is best assessed in the individual patient by therapeutic trial.

The real controversy with chemotherapy concerofs $s \overrightarrow{0}$ not which drugs to use but when this treatment shoufdor be used in the course of metastatic disease and in whicho patients. It is often argued that the earlier chemoth-O erapy is used the longer is survival from the start of $\bar{D}$ treatment. This is undoubtedly true but is of coursea axiomatic and represents a gross example of the pitfall of lead-time bias (Chamberlain, 1982). Given the potentially long natural history of the disease, mean-을 ingful comparisons of survival can only be made from the time of first diagnosis of metastases and not from the entirely arbitrary point of intervention with chemotherapy. However obvious this might seem, such information has very rarely been provided until: quite recently in chemotherapy studies and even now is 3 . given only in the minority of published papers.

A further pitfall concerns the constant finding that responders to chemotherapy live longer than non-o responders; this is commonly put forward as evidence that chemotherapy prolongs survival. Again this is a fallacious argument: response to chemotherapy might simply represent an inherent feature of biologicallyo 'good-prognosis' disease and reflect a sub-group of 0 patients who would live longer anyway. Furthermore N it is possible that non-responders might actually have their survival shortened by the toxicity of ineffective treatment. This is not merely fanciful: ineffective but $\frac{0}{\Phi}$ toxic chemotherapy has been shown in randomised $\stackrel{\oplus}{\longrightarrow}$ trials to shorten survival in other tumour types 0 (Landgren et al., 1973; Brunner et al., 1973). 
The most convincing way to investigate the contribution of chemotherapy to survival would be through a randomised trial with a no-treatment control arm. This has never been carried out for patients with metastatic breast cancer. However, in one study, survival in a group of patients treated with combination chemotherapy at the Royal Marsden Hospital was compared to that of an earlier similar group which never received chemotherapy simply because it was not available at that time. No significant overall survival difference was seen between the two groups, and indeed the chemotherapy-treated patients did rather worse (Powles et al., 1980). A subsequent similar Canadian study tended to support these findings, with no significant increase in survival either from diagnosis or first metastases associated with the advent of combination chemotherapy in the mid 1970s (Paterson et al., 1982).

These studies present a real paradox. Clinicians treating this disease have all seen patients critically ill with life-threatening liver or lung metastases responding to chemotherapy, sometimes dramatically, and going on to survive in good health for many months. Such patients undoubtedly have their survival prolonged by treatment. The explanation for this paradox may rest in the lack of patient selection for chemotherapy and the tendency at that time (certainly in the Royal Marsden study) to treat most patients with chemotherapy early in the course of their metastatic disease, often before a trial of endocrine therapy and often continuously throughout the course of their illness. This is no longer our policy, and in retrospect it seems very possible that no overall survival benefit was seen because the advantages of this approach for some patients were out-weighed by the disadvantages of toxic therapy for many others without life-threatening disease who might have done better initially on endocrine treatment or indeed no treatment.

An important recent Swiss trial supports this hypothesis. Patients were randomised to receive either immediate chemotherapy given concurrently with endocrine therapy or else to have endocrine therapy alone, keeping chemotherapy in reserve (Cavalli et al., 1983). No significant survival benefit was seen either for pre-menopausal or post-menopausal patients treated initially with chemotherapy and endocrine therapy. More important, in a large sub-group of postmenopausal patients who had so called 'good-prognosis' disease with a slow clinical tempo, survival was very significantly reduced in patients receiving initial chemotherapy concurrently with endocrine therapy, indicating that for this group such an approach was not merely without benefit but was positively harmful.

Do any sub-groups achieve survival benefit from chemotherapy? There is some support for the hypothesis that patients with life-threatening disease proba- bly do. In a trial comparing combination chemotherapy (cyclophosphamide, methotrexate and 5fluorouracil) with single agent melphalan. $53 \%$ of patients responded to the combination whereas only $20 \%$ responded to melphalan. Indeed, oral melphalan would be considered a very ineffective therapy for metastatic breast cancer today. Despite these differences in response rate, survival differences were minor (12 months versus 9 months). However, one group of patients did show a significant survival benefit after combination chemotherapy, namely those with liver metastases (Canellos et al., 1976). Similar findings emerge from another trial in which a 5 drug combination was compared to sequential single agent treatment. The former (and fairly conventional) therapy achieved a response rate of $56 \%$ compared with only $32 \%$ for the latter (conventionally considered unorthodox and fairly ineffective approach). Again. however, this difference in response rate was translated into a survival benefit for chemotherapy only in patients with liver metastases; patients without liver metastases had the same median survival whichever therapy was used (Chlebowski et al., 1979).

\section{Current approach to the medical management of advanced breast cancer}

All these observations argue for a conservative overall approach to the management of advanced breast cancer with treatment choices determined mainly by disease tempo and to a lesser extent sites of involvement.

For most patients an initial trial of endocrine therapy is indicated and current evidence suggests that tamoxifen is as effective as any other treatment and has the lowest incidence of side effects. It is not necessary to know the cytoplasmic oestrogen or progesterone receptor status of the tumour before starting tamoxifen; a therapeutic trial is simpler, cheaper and more accurate. In patients responding to tamoxifen, second line endocrine therapy should be considered at relapse.

Chemotherapy is indicated as first line treatment only in patients with rapidly progressive metastatic disease occurring after a short tumour-free interval, and particularly when this involves critical visceral sites including liver; here significant survival benefit may be achieved. Otherwise, because of its greater toxicity, chemotherapy is best reserved until metastases are clearly progressing despite endocrine treatment. Again this should be given as a therapeutic trial and stopped after 2 courses if there is no evidence of palliative benefit. Finally and perhaps most important, patients with slow tempo tumour may do best on no specific treatment at all during asymptomatic periods in the course of their disease. 


\section{Adjuvant therapy}

The rationale for adjuvant therapy in breast cancer is based on two premises. The first is that most patients presenting with clinically localised disease already have metastatic spread, albeit undetectable, from which they will eventually die. The evidence to support this premise is very strong and is based on the inability of local treatment, however radical, to make any major influence on survival. The second premise is that chemotherapy or endocrine therapy, which can only achieve transient tumour regression for clinically overt metastases, might be more effective in achieving prolonged survival or cure if given early in the clinical course of the disease as an adjuvant to surgery. The evidence to support this premise is more tenuous: certainly it has been well demonstrated in experimental animal tumours that curability with chemotherapy is related to low total tumour burden; likewise in the clinic adjuvant chemotherapy has been shown to increase the cure rate significantly for some paediatric tumours including in particular nephroblastoma. However, both these examples are based on tumour types which are much more sensitive to chemotherapy than breast carcinoma.

The earliest clinical trials of adjuvant chemotherapy in breast cancer date back more than 25 years (Fisher et al., 1968). However, the real stimulus to current interest in this approach came from the initial results of two randomised trials published in the mid 1970s. Both studied patients with axillary node involvement and both had no-treatment control arms. The first, a multicentre American trial, studied the effect of a two year course of single agent melphalan (Fisher et al., 1975), and the second, based in Milan, used a combination of cyclophosphamide, methotrexate and 5fluorouracil (CMF) for 12 months (Bonadonna et al., 1976). Both these trials showed a delay in tumour recurrence for patients treated with chemotherapy. Although the follow up time was very short (a maximum of 18 and 27 months) and although no survival data were given, these results were hailed as a major advance in the treatment of breast cancer, first in the medical literature (Holland, 1976) and subsequently in the lay press. Adjuvant chemotherapy immediately became the treatment of choice for many thousands of women with early breast cancer, particularly in the USA.

In the euphoria an unfortunate decision was made that was to prejudice the interpretation of all subsequent American trials: no-treatment control arms were considered 'unethical' and were dropped. In contrast, a more cautious approach was adopted in the UK where several no-treatment control trials were initiated. With the passage of time these have taken on increasing importance.

What has happened in the interim? With follow up, no clear-cut survival advantage in the melphalan trial has been published, and a combined Guy's Hospital- 3 Manchester trial repeating the melphalan study has $\stackrel{\varnothing}{\complement}$ likewise failed to show any significant survival dif $-\stackrel{c}{.}$ ferences or even any significant delay in tumour $\overrightarrow{\bar{B}}$ recurrence (Rubens et al., 1983). The Milan CMF trial continues to show some promise and the predicted 8 등 year survival results, so far presented only in sum- $\frac{\bar{c}}{\bar{\omega}}$ mary, suggest a survival advantage in pre-menopausal $\vec{\Phi}$ patients of $65 \%$ versus $45 \%$ for untreated controls (Bonadonna et al., 1983). No survival difference क whatever has emerged for post-menopausal patients. $\vec{O}$ Although this difference is encouraging, it should be noted that $80 \%$ of pre-menopausal patients and over $\bar{\sigma}$ $90 \%$ of all patients given 12 months combination: chemotherapy failed to achieve any benefit. A further 3 cause for caution is that subsequent similar trials are so far failing to confirm these findings. The Guy'sManchester group have repeated the CMF trial but $\vec{N}$ have so far failed to demonstrate any significant survival advantage, albeit with shorter follow up $\vec{\nabla}$ (Howell, 1983). A delay in time to tumour recurrence $ᄋ$ has been established but only for pre-menopausal patients. Likewise, a West Midlands multicentre 7 group have compared a 5 drug adriamycin-containing $\frac{D}{5}$ regimen against no treatment and have failed to demonstrate survival benefit (J.M. Morrison, personal communication). Other trials also failing $\vec{\varphi}$ show survival benefit for adjuvant chemotheraf include a British multicentre group using a $4 \operatorname{dr}$ combination (Bates, 1983) and a King's College Hospital trial using oral melphalan and methotrexate (M. Baum, personal communication).

In the absence so far of real survival benefit from $\frac{\mathscr{Q}}{\varnothing}$ many trials, the parameter of delay in time to tumour $\cong$ recurrence has been widely claimed as the basis for so- $\overrightarrow{\overrightarrow{0}}$ called 'benefit' with adjuvant chemotherapy. This is 3 frequently referred to as 'relapse free survival', a potentially misleading term which does not in itself say anything about real survival benefit. It is scarcely 윽 surprising that chemotherapy which achieves temporary tumour growth delay in most patients with $\frac{5}{3}$ overt clinical metastases would do the same when the disease is subclinical, and the enthusiasm generated by $\frac{\circ}{3}$ trials showing a delay in tumour recurrence is hard to understand and certainly of little biological interest. $\mathrm{A}$ 을 delay in time to tumour recurrence is of no true $\frac{D}{0}$ survival benefit if it is bought at the expense of a shorter interval between relapse and death, and indeed $N$ this has already been demonstrated for some trials of $N$ both endocrine therapy and chemotherapy (Cole, N 1970; Chlebowski et al., 1981). In such circumstances, $\frac{\omega}{\sigma}$ there are arguments in favour of withholding treatment until relapse: its clinical effectiveness can then be $\stackrel{\varrho}{\simeq}$ assessed directly in terms of tumour response and $\stackrel{\Phi}{\rightarrow}$ patients already cured by the original local treatment $\stackrel{?}{?}$ are spared unnecessary further systemic treatment 
associated with toxicity and uncertain long term sequelae.

There is perhaps one argument in favour of treatment which delays time to recurrence and that is that the psychological blow of recurrence is postponed. This blow can undoubtedly be a severe one, but this argument must be weighed against problems associated with treatment itself. The first of these concerns morbidity. For simple forms of adjuvant endocrine therapy including for example tamoxifen (see below) morbidity appears very minor, but for adjuvant chemotherapy several studies have demonstrated that the side effects and toxicity are associated with a significant deterioration in quality of life and psychiatric morbidity (Palmer et al., 1980; Wilcox et al., 1982; Maguire et al., 1980; McCardle et al., 1981). Indeed, in one study, almost one third of patients could not continue treatment presumably despite being told that it might prolong life (Wilcox et al., 1982) and in another, a similar proportion of patients volunteered that the experience could never be gone through again (Palmer et al., 1980). Quite apart from the psychological problems associated with treatment morbidity, it could be argued that the psychological blow of relapse despite having undergone prolonged intensive therapy is even more devastating than without such treatment, and indeed this has been my own clinical experience on several occasions. There are no clear-cut answers to this difficult question and in the end value judgements are required; but many of us feel that the short term psychological gain of delayed recurrence in a small minority of patients cannot balance against the morbidity and ineffectiveness of toxic therapy for the majority.

Is there a role for less toxic forms of adjuvant therapy? First, it is possible that less prolonged chemotherapy may be as effective. In a second Milan trial, survival after 6 courses of CMF chemotherapy appears as good as after 12 (Bonadonna et al., 1983). More strikingly, an early Norwegian trial with 15 year follow up has shown a persisting $10 \%$ survival advantage for patients randomised to receive a single 6 day course of cyclophosphamide, immediately after surgery (Nissen-Meyer $e t$ al., 1982). Of interest is the fact that this difference only began to emerge 4 years after treatment; given the natural history of breast cancer, this observation would be consistent with true eradication of minimal micro-metastatic disease rather than transient growth delay in subclinical but relatively large metastases. This important trial is currently being repeated and further trials on short term chemotherapy are clearly indicated.

Finally, the observation that most chemotherapy trials have shown delays in tumour recurrence predominently in pre-menopausal patients suggests the possibility that these drugs may be acting at least partly through an endocrine effect. Certainly the majority of pre-menopausal patients on combination chemotherapy develop amenorrhoea (Bonadonna $e t$ $a l ., 1977)$ and it is now well established that these drugs suppress ovarian function with a significant fall in circulating plasma oestrogens and a large rise in follicle stimulating and luteinizing hormone (Rose \& Davis, 1980). This has stimulated a generation of new adjuvant endocrine therapy trials, most of which are based on tamoxifen. Already a large multicentre randomised trial in the UK is showing a significant survival advantage for patients treated with tamoxifen compared with no-treatment controls. This advantage is seen both in pre- and post-menopausal patients; most interestingly at present it is also seen in oestrogen receptor positive and receptor negative patients $(M$. Baum, personal communication). As with adjuvant chemotherapy, only a minority of patients appear to achieve benefit. However, the great advantage of tamoxifen over chemotherapy is its lack of side effects and toxicity. Until new and more effective drugs become available, this approach would therefore seem one of the more promising in adjuvant systemic therapy.

\section{Adjuvant systemic therapy: Conclusions}

Adjuvant chemotherapy consistently delays the time to tumour recurrence, at least in premenopausal patients, but so far only one modern trial has shown any significant survival benefit, and again only in premenopausal patients. Toxicity and morbidity can be considerable, and this type of chemotherapy must continue to be considered experimental until more convincing survival benefits emerge. Current data suggest that it is entirely contra-indicated in postmenopausal patients. There is some evidence to suggest that adjuvant endocrine therapy or very short duration chemotherapy may also confer some survival advantage. The relative lack of toxicity of these latter approaches make them attractive for further investigation.

\section{References}

BATES, T. (April, 1983). Data presented at symposium: 'Treatment decisions in early breast cancer' Royal College of Physicians of London.

BLOOM, H.J.G., RICHARDSON, W.W. \& HARRIES, E.J.

(1962). Natural history of untreated breast cancer (1805-1933): comparison of untreated and treated cases according to histological grade of malignancy. British Medical Journal, 2, 213. 
BONADONNA, G., BRUSAMOLINO, E., VALAGUSSA, P., ROSSI, A., BRUGNATELLI, L., BRAMBILLA, C., DE LENA, M., TANCINI, G., BAJETTA, E., MUSUMECI, R. \& VERONESI, U. (1976). Combination chemotherapy as an adjuvant treatment in operable breast cancer. New England Journal of Medicine, 294, 405.

BONADONNA, G., ROSSI, A., VALAGUSSA, P., BANFI, A., \& VERONESI, U. (1977). The CMF programme for operable breast cancer with positive axillary nodes. Cancer, 39, 2904.

BONADONNA, G., ROSSI, A., TANCINI, G., \& VALAGUSSA, P. (1983). Adjuvant chemotherapy in breast cancer. Lancet, $\mathbf{i}$, 1157.

BRINKLEY, D. \& HAYBITTLE, J.L. (1975). The curability of breast cancer. Lancet, ii, 95.

BRUNNER, K.W., MARTHALER, T. \& MULLERM, W. (1973). Effects of long term adjuvant chemotherapy with cyclophosphamide for radically resected bronchogenic carcinoma. Cancer Chemotherapy Reports, 4, 125.

CANELLOS, G.P., POCOCK, S.J., TAYLOR, S.G., SEARS, M.E., KLASSEN, D.J. \& BAUM, P.R. (1976). Combination chemotherapy for metastatic breast carcinoma. Cancer, 38, 1882.

CAVALLI, F., BEER, M., MARTZ, G., JUNGI, W.F., ALBERTO, P., OBRECHT, J.P., MERMILLOD, B., \& BRUNNER, K.W. (1983). Concurrent or sequential use of cytotoxic chemotherapy and hormone treatment in advanced breast cancer. British Medical Journal, 286, 5.

CHAMBERLAIN, J. (1982). Screening for cancer. British Journal of Hospital Medicine, 27, 583.

CHLEBOWSKI, R.T., IRVIN, L.E., PUGH, R.P., SADOFF, L., HESTAFF, R., WIENER, J.M. \& BATEMAN, J.R. (1979). Survival of patients with metastatic breast cancer treated with either combination or sequential chemotherapy. Cancer Research, 39, 4503.

CHLEBOWSKI, R.T., WEINER, J.M., LUCE, J., HESTORFF, R., LANG, J.E., REYNOLDS, R., GODFREY, T., RYDEN, V.M.J. \& BATEMAN, J.R. (1981). Significance of relapse after adjuvant treatment with combination chemotherapy or 5fluorouracil alone in high-risk breast cancer. A Western Cancer Study Group project. Cancer Research, 41, 4399.

COLE, M.(1970). Prophylactic compared with therapeutic Xray artificial menopause. In Second Tenovus workshop on breast cancer, Joslin, C.A.F., Gleave, E.N., (eds), p. 2-11. Cardiff: Alpha Omega Alpha Publishing Co.

FISHER, B., RAVDIN, R.G., AUSMAN, R.K., SLACK, N.H., MOORE, G.E. \& NOER, R.J. (1968). Surgical adjuvant chemotherapy in cancer of the breast: results of a decade of cooperative investigation. Annals of Surgery, 168, 337.

FISHER, B., CARBONE, P., ECONOMOU, S.G., FRELICK, R., GLASS, A., LERNER, H., REDMOND, C., ZELEN, M., BAND, P., KATRYCH, D.L., WOLMARK, N. \& FISHER, E.R. (1975). 1-Phenylalanine mustard (1-PAM) in the management of primary breast cancer; a report of early findings. New England Journal of Medicine, 292, 117.

HARRIS, A.L., POWLES, T.J. \& SMITH, I.E. (1982). Aminoglutethimide in the treatment of advanced postmenopausal breast cancer, Cancer Research, 42, 3405.

HENDERSON, I.C. \& CANELLOS, G.P. (1980). Cancer of the breast: the past decade (2). New England Journal of Medicine, 302, 78.
HOLLAND, J.F. (1976). Major advance in breast cancer therapy. New England Journal of Medicine, 294, 440.

HOWELL, A. (1983). Data presented at symposium: 'Treatment decisions in early breast cancer' Royal College of Physicians of London.

KIANG, D.T. (1981). Breast Cancer: Methods and Results of Endocrine Therapy. In Hormonal Management of Endocrine-Related Cancer, Stoll, B.A., (ed), p. 64. LloydLuke: London.

LANDGREN, R.C., HUSSEY, D.H. \& SAMUELS, M.L. (1973). A randomised study comparing irradiation alone to irradiation plus procarbazine in inoperable bronchogenic carcinoma. Radiology, 108, 403.

MAGUIRE, G.P., TAIT, A., BROOKE, M., THOMAS, C., HOWAT, J.M.T., SELLWOOD, R.A. \& BUSH, H. (1980). Psychiatric morbidity and physical toxicity associated with adjuvant chemotherapy after mastectomy. British Medical Journal, 281, 1179.

MARGREITER, R., \& WEIGELE, J. (1984). Tamoxifen for premenopausal patients with advanced breast cancer. Breast Cancer Research and Treatment, 4, 45.

MCARDLE, C.S., CALMAN, K.C., COOPER, A.F., HUGHSON, A.V.M., RUSSELL, A.R. \& SMITH, D.C. (1981). The social, emotional and financial implications of adjuvant chemotherapy in breast cancer. British Journal of Surgery, 68, 261.

NISSEN-MEYER, R., KJELLGREN, K. \& MANSSON, B. (1982). Adjuvant chemotherapy in breast cancer. Recent Results in Cancer Research, 80, 142.

PALMER, B.V., WALSH, G.A., McKINNA, J.A. \& GREENING, W.P. (1980). Adjuvant chemotherapy for breast cancerside effects and quality of life. British Medical Journal, 281 1594.

PATERSON, A.H.G., SZAFRAN, O., CORNISH, F., LEES, A.Wö \& HANSON, J. (1982). Effect of chemotherapy on survivał in advanced breast cancer. Breast Cancer Research and Treatment, 1, 357.

POWLES, T.J., COOMBES, R.C., SMITH, I.E., FORD, H.T. \& GAZET, J-C. (1980). Failure of chemotherapy to prolong survival in a group of patients with metastatic breast cancer. Lancet, i, 580.

PRIESTMAN, T., BAUM, M., JONES, V. \& FARBES, J. (1977). Comparative trial of endocrine versus cytotoxic treatment in advanced breast cancer. British Medical Journal, 1, 1248.

ROSE, D.P. \& DAVIS, T.E. (1980). Effects of adjuvant chemohormonal therapy on the ovarian and adrenal function of breast cancer patients. Cancer Research, 40, 4043.

RUBENS, R.D., HAYWARD, J.L., KNIGHT, R.K., FENTIMAN, I.S., HOWELL, A., CROWTHER, D., GEORGE, W.D., BULBROOK, R.D., CHAUDARY, M., BUSH, H., SELLWOOD, R.A. \& HOWAT, J.M.T. (1983). Controlled trial of adjuvant chemotherapy with melphalan for breast cancer. Lancet, $\mathbf{i}$, 839.

WILCOX, P.M., FETTING, J.H., NETTESHEIM, K.M. \& ABELOFF, M.D. (1982). Anticipatory vomiting in women receiving cyclophosphamide, methotrexate, and 5-FU (CMF) adjuvant chemotherapy for breast carcinoma. Cancer Treatment Reports, 66, 1601. 